\title{
Second Generation SBAS - Performance Analysis and Bridging Positioning and Integrity Monitoring during SBAS Outages in the Urban Environment
}

\author{
Ahmed El-Mowafy and Kan Wang \\ School of Earth and Planetary Sciences, Curtin University, Australia
}

\section{BIOGRAPHIES}

\begin{abstract}
Ahmed El-Mowafy is Assoc. Professor of Positioning and Navigation and Director of Graduate Research, School of Earth and Planetary Sciences, Curtin University, Australia. He obtained his Ph.D. from the University of Calgary, Canada. He has extensive publications in precise positioning and navigation using GNSS, quality control, integrity monitoring and estimation theory.

Kan Wang is research associate in School of Earth and Planetary Sciences, Curtin University. She received her PhD in GNSS advanced modeling from ETH Zurich in 2016. Her research interest includes SBAS, satellite clock modeling, multi-frequency and multi-GNSS ambiguity resolution, RTK and PPP-RTK processing.
\end{abstract}

\begin{abstract}
Improved SBAS is expected to be a primary positioning method for many land applications such as Intelligent Transport Systems (ITS). In 2017, a second-generation SBAS test-bed was initiated in Australia and New-Zealand in preparation for building an operational system. In addition to the traditional L1 legacy SBAS signals, the Australian/NZ SBAS provides dual-frequency multiconstellation (DFMC) SBAS signals and SBAS-based PPP service for GPS and Galileo observations. This article addresses the use of SBAS in land applications from three important aspects. Firstly, the performance of the different SBAS solution methods is assessed in two main applications, transportation and mining. Tests were conducted under various environments, including open sky, low- and high-density urban, and mining. Performance analysis showed that SBAS solutions provide better positioning than single point positioning, and the DFMC SBAS solutions have better precision than L1 SBAS solutions. Furthermore, SBAS-based PPP solutions delivered a few-dm accuracy, which is a bit worse than traditional PPP with corrections received via the Internet. It is shown that SBAS performance is strongly dependent on the application environment. Secondly, tests show that SBAS outages may occur in urban areas, thus, the paper proposed prediction of the SBAS orbit and clock corrections as time series to enable positioning. The orbits are predicted using Holt-Winters' method and clock corrections were predicted using a second order polynomial with sinusoidal components. A position error due to prediction grow with time, but was less than $0.26 \mathrm{~m}$ after 15 minutes of prediction. Finally, the impact of SBAS outages on computation of the protection levels (PL) needed for integrity monitoring of the new DFMC SBAS is illustrated. It is shown that Horizontal-PLs can reach more than $10 \mathrm{~m}$, which is not suitable for ITS when the previous SBAS parameters before the outage were used, and thus methods are proposed for future research.
\end{abstract}

\section{INTRODUCTION}

The Satellite-Based Augmentation System (SBAS) are used in different regions to augment Global Navigation Satellite System observations to improve positioning and navigation of single point positioning (SPP). SBAS uses geostationary (GEO) satellites to transmit GNSS satellite orbit and clock corrections, regional ionospheric corrections, ranging and integrity information. Currently operational SBAS includes the USA Wide Area Augmentation System (WAAS), the European Geostationary Navigation Overlay Service (EGNOS), the Japanese Multi-functional Satellite Augmentation System (MSAS), the Russian System for Differential Correction and Monitoring (SDCM) and the Indian GPS-aided GEO Augmented Navigation (GAGAN). These systems, except for MSAS, currently support only single-frequency measurement users but in the future, such as for EGNOS V3 will include dualfrequency multi-constellation (DFMC) service. 
Since September 2017, Australia and New Zealand (referred to as AUS/NZ) commenced SBAS test-bed over the Asia-Pacific and Australasia area [1]. This SBAS comprises a second-generation SBAS service in a pre-step to building a fully operational system within a few years. The test-bed provides in addition to the L1 legacy SBAS signals that are transmitted over L1, the DFMC SBAS corrections transmitted over L5. L1 SBS meets the RTCA/DO-229 standards and is open to current mass-market receivers implementing SBAS positioning mode. The second service; the new generation DFMC SBAS L5 prototype signal, complies with the specification defined in WG62 GAL GPS SBAS MOPS v0.6.1 [2]. The WG62 is a draft standard that defines future DFMC services. Due to bandwidth limitations, the AUS/NZ DFMC service only supports GPS and Galileo satellites for which corrections and integrity data are available [3]. While the L1 SBAS service transmits range, orbit, clock and ionospheric delay corrections, applicable to the service area covering the complete Australian and New Zealand territories, the DFMC SBAS L5 provides GPS and Galileo dual-frequency augmentation that can be used anywhere across the whole footprint of the GEO satellite. An Inmarsat 4F1 GEO satellite is used, which is located at longitude of 143.4 degrees East [1].

The AUS/NZ SBAS additionally broadcast precise satellite orbits and clock corrections to support real-time float-ambiguity Precise Point Positioning (PPP) service that can deliver 5-20 cm accuracy $[4,5,6]$. The corrections are transmitted over L1 frequency for GPS only and L5 for GPS+Galileo. This service allows for superior availability of PPP compared with traditional PPP. It enables PPP anywhere in the footprint of the SBAS GEO satellite, i.e. most Australia-Asia pacific region, whereas traditional PPP depends on the Internet to receive the corrections, and thus is limited to areas where the Internet is available. Noting the vast size of low populated areas in Australia and the surrounding oceans, such service of providing PPP over satellites brings significant benefits to users in these areas. Preliminary SBAS test-bed messages and positioning results have been reported in [1, 7, 8]. Under good satellite visibility conditions, accuracy as good as sub-m can be obtained when using L1 SBAS and DFMC SBAS, and at sub-decimeter using PPP. Whilst the PPP positioning method has higher accuracy than L1 and DFMC SBAS, it requires a period time to converge and achieve this level of accuracy, which can be in the range of 30-60 minutes [9], whereas L1 SBAS and DFMC SBAS positioning is achieved almost instantaneously due to being code-based.

To complement the above studies, this paper comprises three sections. The first section presents performance analysis of the AUS/NZ second generation (L1, DFMC) SBAS and PPP positioning with a focus on its use for advanced transport applications and in mining. The outcomes of this research can assist in clarifying the potential of different services of SBAS in improving the safety and efficiency of the road transport in Australia and mining applications. Since transport applications experience continuous changes in the operational environment, the analysis is conducted classifying the work environment into three classes: suburban, low -density urban, and high-density urban. Tests are described and results are presented and analysed. Performance metrics such as precision, accuracy, and availability are discussed.

Unlike the application of SBAS in aviation where SBAS is almost available all the time, in land applications SBAS signals may suffer from outages due to obstruction of the signals by surrounding structures. Therefore, the second part of the paper proposes a method to bridge such short outage in SBAS data, where the satellite orbit and clock corrections are predicted. Finally, the third section of the paper shows the impact of these SBAS outages, with special focus on the new DFMC SBAS, on computation of the horizontal protection levels (HPLs), needed for integrity monitoring (IM). The study highlights the needed for methods that can be used during this outage for integrity monitoring consistent with SBAS (IM). These methods will be covered in a follow up paper.

\section{PERFORMANCE OF THE SECOND-GENERATION SBAS}

In this section, the performance of the $2^{\text {nd }}$ generation AUS/NZ SBAS is demonstrated, where three types of SBAS-aided solutions are analysed and compared, namely:

- $\quad$ The L1 SBAS solutions, which use the GPS L1 and the Galileo E1 signals, transmitted over L1.

- $\quad$ The DFMC SBAS solutions, make use of the GPS L1+L2 and Galileo E1+E5a signals, sent over L5.

- $\quad$ The SBAS-aided PPP utilizes also the GPS L1+L2 and Galileo E1+E5a measurements, sent over L5.

- The GPS L1 single point positioning (SPP) results are also processed to show the benefit of SBAS when augmenting SPP.

For dual-frequency users using DFMC SBAS or PPP solutions, the first-order ionospheric delays is eliminated by forming the ionosphere-free linear combination of the observations. The GPS L2 signals were used due to the current limited number of GPS IIF satellite sending L5 signals. However, switching from L2 to L5 is done in April 2019. With the increasing number of GPS III and Galileo satellites, as well as the improvement of the technologies in both hardware and software, better positioning results is expected. 


\section{Description of the SBAS performance tests}

To demonstrate the SBAS positioning results under different measurement conditions, experiments were performed in kinematic tests in Wollongong, Sydney and at a mine site in Western Australia. The measurement environments are distributed into five categories that include an open-sky scenario, an area surrounded by tress, a low-density urban area, a central business district (CBD), and an open-pit mine site. The tests in Wollongong and Sydney were performed using a vehicle, shown in Figure1. A Leica AR10 antenna was mounted on top of the vehicle, together with a Septentrio AsteRx-U receiver tracking the GNSS data and SRX-10 decoding and logging the SBAS messages [10]. The PPP results was provided in real-time by the magicAPK software, and in postmission mode, the SPP, the L1 and DFMC SBAS data were processed with the magicGEMINI software (https://www.gmv.com/en/Products/magicGEMINI/) using the same code and phase observations. The experiments in Wollongong and in Sydney were performed in June and April 2018, respectively.

The experiment at the mine site was performed on November $4^{\text {th }}, 2018$. A Topcon G3-A1 antenna was mounted on a drill machine (see Figure 1b) and connected with a magicUT receiver [10]. The magicUT is capable of providing either the L1 SBAS, DFMC SBAS or PPP solutions in real time. Therefore, the PPP was processed in real time and using the collected GNSS data and the SBAS corrections, the SPP, L1 SBAS and DFMC SBAS solutions were processed in post-mission using the magicGEMINI software. The test locations, test environments, test days, test platforms, and the hardware and the software used for both the transportation and mining experiments are summarized in Table 1. The set A corresponds to Septentrio AsteRx-U receiver, Leica AR10 antenna and SRX-10, and the set B corresponds to the Topcon G3-A1 antenna and the magicUT receiver.

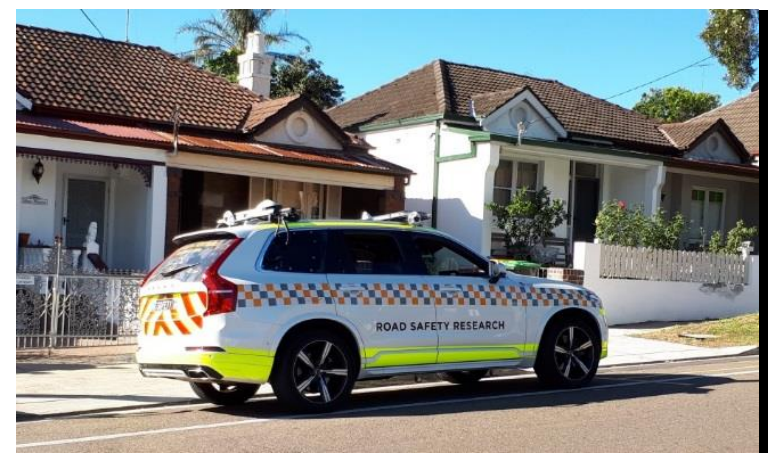

(a)

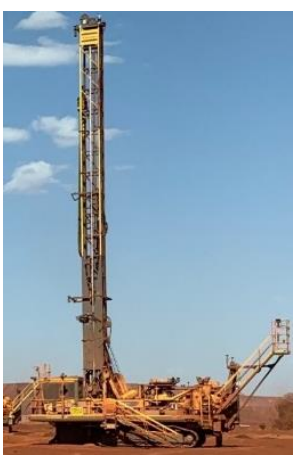

(b)

Fig 1. Test vehicle in Wollongong and Sydney (a), and the drill machine used for the test at the mine site (b)

Table 1. Descriptions of the kinematic experiments used for SBAS analysis

\begin{tabular}{|c|c|c|c|c|c|c|}
\hline Test No. & Location & Environment & Test day & Platform & Hardware & Software \\
\hline 1 & Wollongong & Open-sky & June 12018 & Vehicle & Set A & $\begin{array}{l}\text { magicAPK, } \\
\text { magicGEMINI }\end{array}$ \\
\hline 2 & Wollongong & $\begin{array}{l}\text { Area surrounded by } \\
\text { trees }\end{array}$ & June 12018 & Vehicle & Set $\mathrm{A}$ & $\begin{array}{l}\text { magicAPK, } \\
\text { magicGEMINI }\end{array}$ \\
\hline 3 & Sydney & $\begin{array}{l}\text { Low-density urban } \\
\text { area }\end{array}$ & April 202018 & Vehicle & Set A & $\begin{array}{l}\text { magicAPK, } \\
\text { magicGEMINI }\end{array}$ \\
\hline 4 & Sydney & CBD & April 242018 & Vehicle & Set $\mathrm{A}$ & $\begin{array}{l}\text { magicAPK, } \\
\text { magicGEMINI }\end{array}$ \\
\hline 5 & Roy Hill & Mine site & November 42018 & Drill machine & Set B & magicGEMINI \\
\hline
\end{tabular}

\section{Strategy of the analysis}

In this study, only the horizontal positioning solutions are of concern and are analysed. The easting and northing coordinates were generated using the Universal Transverse Mercator (UTM) system and the Geocentric datum of Australia 1994 (GDA94). They were compared with the ground truth, which was obtained through independent relative kinematic positioning processing the rover data with data from a reference station located in the same region, with a baseline length within $9 \mathrm{~km}$. 
The data analysis first goes through an outlier detection. Time epochs with easting or northing errors exceeding $\pm 10 \mathrm{~m}$ are screened out for the SPP and SBAS solutions. For PPP, the threshold decreases to $\pm 5 \mathrm{~m}$. Afterwards, epochs with outliers outside a region of the mean value \pm triple the standard deviations (STD) in the east or north direction are eliminated. The solutions from different SBAS modes were compared in terms of the mean of absolute positioning errors, STDs, the root mean squares (RMS) of the positioning errors, solution availability and outlier rates as performance metrics. The availability is calculated as the ratio of the number of time epochs with valid solutions with respect to the total number of time epochs that have valid observations. The outlier rate is computed as the number of epochs removed during the outlier detection process divided by the epochs having valid solutions.

\section{Test 1: Results of the Open-sky environment}

The first kinematic test was conducted in June $1^{\text {st }}, 2018$, from 0:00 to 2:10 in GPS time (GPST) in Wollongong, Australia under an open-sky scenario. The positioning errors in the east and north directions are shown in Figure 2a. It can be observed that positioning errors of the PPP solutions have an obviously smaller range of errors than those of the other solution types, while no significant differences are observed between the SPP, L1 SBAS and DFMC SBAS solutions in this test with a mean error around 1 $\mathrm{m}$. The mean absolute errors and standard deviations of the positioning errors are further illustrated in Figure $2 \mathrm{~b}$ and the performance metrics are given in Table 2. From Table 2, a high solution availability of 99\% for PPP can be observed. The L1 SBAS and DFMC solutions are also available in about $95 \%$ of the time. In addition, a larger outlier rate can be observed in the SPP case than that in the SBAS-aided cases.

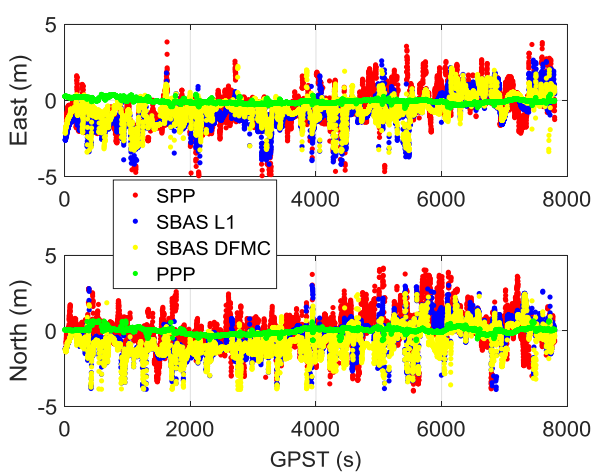

(a)
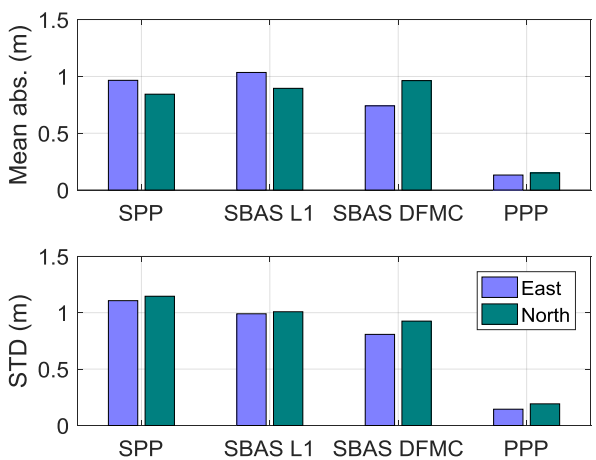

(b)

Fig 2. East and north positioning errors (a), the mean absolute errors and STD of the errors (b) for Test 1 in open-sky scenario

Table 2. Statistics of the positioning errors for Test 1 in open-sky scenario

\begin{tabular}{ccccc}
\hline Parameters & SPP & L1 SBAS & DFMC SBAS & PPP \\
\hline Mean abs. error (East) (m) & 0.97 & 1.04 & 0.74 & 0.13 \\
\hline Mean abs. error (North) (m) & 0.84 & 0.90 & 0.96 & 0.15 \\
\hline STD (East) (m) & 1.11 & 0.99 & 0.81 & 0.14 \\
\hline STD (North) (m) & 1.15 & 1.01 & 0.93 & 0.19 \\
\hline RMS (East) (m) & 1.19 & 1.24 & 0.95 & 0.16 \\
\hline RMS (North) (m) & 1.15 & 1.11 & 1.17 & 0.19 \\
\hline Availability (solutions) & $87 \%$ & $95 \%$ & $95 \%$ & $99 \%$ \\
\hline Outlier rate & $6 \%$ & $3 \%$ & $3 \%$ & $2 \%$ \\
\hline
\end{tabular}

\section{Test 2: Results of the Area surrounded by trees}

The second kinematic test was performed in Wollongong in an area characterized by the presence of trees on the sides of a narrow road for two different time periods in the same day, i.e., from 06:20 to 07:00 and from 07:16 to 07:40 in GPST. The east and north positioning errors are illustrated in Figure 3a. Note that between $23596 \mathrm{~s}$ and 23697 s no GNSS raw observations were tracked by the receiver. Due to the worse measurement conditions compared to Test 1, the PPP solutions were sometimes not able to be generated, which resulted in a worse availability of the solutions compared to that in the open-sky scenario. The positioning errors have sometimes also reached several meters in the PPP case due to not reaching convergence. The mean absolute errors, STD and 
RMS of the east and north errors are further shown in Figure $3 \mathrm{~b}$ and Table 3. The PPP and SBAS solutions provide better precision and less outlier rate than the SPP solutions, while their RMS are still at the meter-level. Moreover, the SBAS DMFC mean error was less than that of L1 SBAS. The availabilities of the solutions have decreased for all solution types compared to the open-sky scenario.

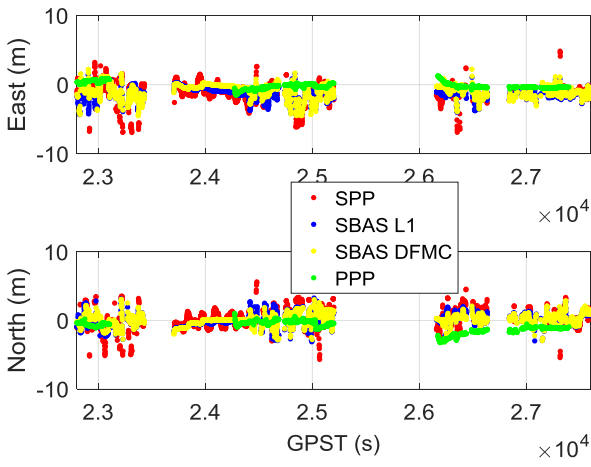

(a)

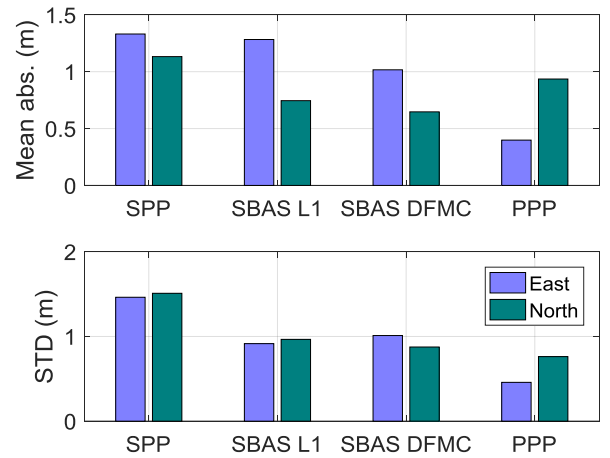

(b)

Fig 3. East and north errors (a), the mean absolute errors and STD of the errors (b) for test in area surrounded by trees

Table 3. Statistics of the positioning errors for Test 2 in area surrounded by trees

\begin{tabular}{ccccc}
\hline Parameters & SPP & L1 SBAS & DFMC SBAS & PPP \\
\hline Mean abs. error (East) $(\mathrm{m})$ & 1.33 & 1.28 & 1.02 & 0.40 \\
\hline Mean abs. error (North) $(\mathrm{m})$ & 1.13 & 0.75 & 0.65 & 0.94 \\
\hline STD (East) $(\mathrm{m})$ & 1.46 & 0.92 & 1.01 & 0.46 \\
\hline STD (North) $(\mathrm{m})$ & 1.51 & 0.97 & 0.88 & 0.76 \\
\hline RMS (East) (m) & 1.83 & 1.53 & 1.36 & 0.49 \\
\hline RMS (North) (m) & 1.52 & 0.97 & 0.88 & 1.19 \\
\hline Availability (solutions) & $54 \%$ & $88 \%$ & $88 \%$ & $65 \%$ \\
\hline Outlier rate & $5 \%$ & $2 \%$ & $3 \%$ & $1 \%$ \\
\hline
\end{tabular}

\section{Test 3: Results of the low-density urban area}

The third experiment was performed on April 202018 in Sydney following the bus route number 333 in an area characterized by low-height buildings for three different time intervals, i.e. from 00:00:00 to 00:18:20, from 00:34:50 to 00:49:30 and from 01:22:20 to 01:38:15 in GPST. The east and north positioning errors are illustrated in Figure 4a, and the statistics are further shown in Figure $4 \mathrm{~b}$ and Table 4. Here a significant increase in the outlier rate is observed in the SPP solutions, which indicates an increasing number of epochs with large positioning errors as shown in the bottom panel of Figure 4a. The SBAS solutions have shown better precision (in terms of the STDs) and accuracy (shown by the mean position error) compared to those of the SPP solutions with a mean $1.2-$ $2 \mathrm{~m}$, while not surprisingly the PPP solutions generally have the best positioning behaviour, but its error has doubled compared with the open-sky case due to observing a less number of satellites. The availabilities of the solutions are larger in the SBAS-aided cases than that in the SPP case and DFMC SBAS outperformed L1 SBAS. This is consistent with the results shown in Tests 1 and 2.

Table 4. Statistics of the positioning errors for Test 3 in low-density urban area

\begin{tabular}{ccccc}
\hline Parameters & SPP & L1 SBAS & DFMC SBAS & PPP \\
\hline Mean abs. error (East) (m) & 1.35 & 1.04 & 0.91 & 0.26 \\
\hline Mean abs. error (North) (m) & 2.87 & 1.96 & 1.82 & 0.34 \\
\hline STD (East) (m) & 1.76 & 1.27 & 1.26 & 0.25 \\
\hline STD (North) (m) & 3.32 & 2.21 & 1.46 & 0.40 \\
\hline RMS (East) (m) & 1.77 & 1.40 & 1.29 & 0.33 \\
\hline RMS (North) (m) & 3.84 & 2.64 & 2.14 & 0.46 \\
\hline Availability (solutions) & $54 \%$ & $81 \%$ & $81 \%$ & $88 \%$ \\
\hline Outlier rate & $15 \%$ & $3 \%$ & $4 \%$ & $4 \%$ \\
\hline
\end{tabular}




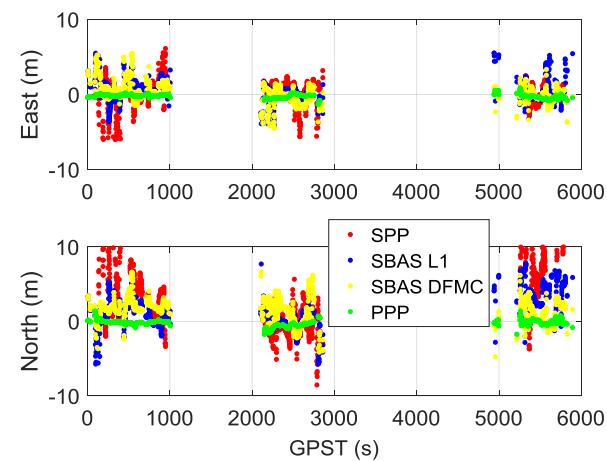

(a)
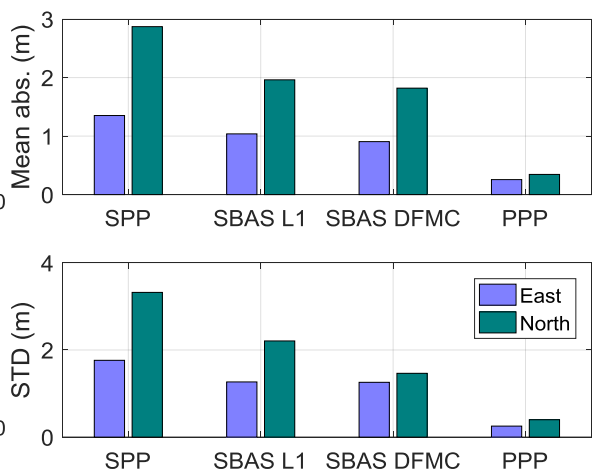

(b)

Fig 4. East and north positioning errors (a), the mean absolute errors and STD of the errors (b) for test in low-density urban area

\section{Test 4: Results of the CBD test}

The fourth test is performed in April 242018 in the CBD area of Sydney from 03:00:38 to 04:30:00 in GPST. The east and north errors are shown in Figure 5a, and their statistics are further depicted in Figure 5b and Table 5. Note that between about $14300 \mathrm{~s}$ and $15300 \mathrm{~s}$, very limited observations were tracked by the GNSS receiver due to signal obstruction by the surrounding buildings, so that the ground truth and most of the solutions cannot be produced. Having a look at Table 5, it can be observed that the precision and accuracy of the PPP solutions are generally at sub-m level. The SBAS solutions have delivered better positioning results than those of the SPP solutions, which are still at meter-level. Compared to the Test 3, larger outlier rates are observed for the L1 SBAS and DFMC solutions. Again, the DFMC SBAS delivered better solution than L1 SBAS. The precision and accuracy of PPP solutions were worse than those in the low-density urban areas.

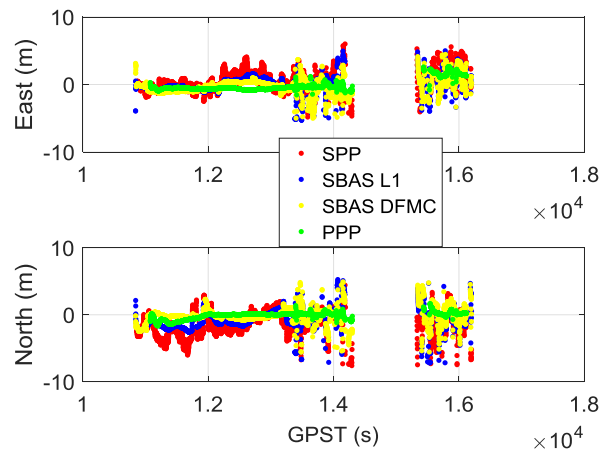

(a)
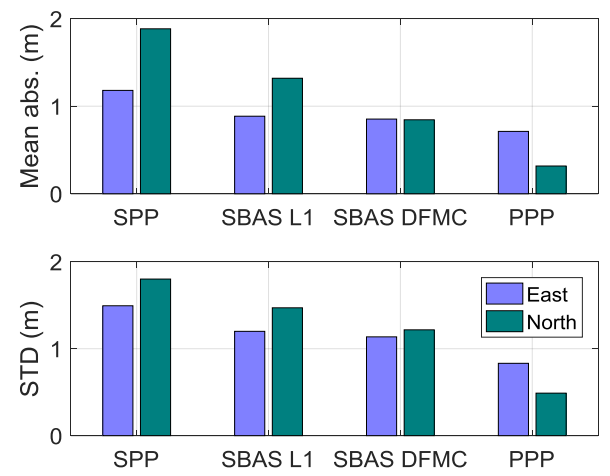

(b)

Fig 5. East and north positioning errors (a), the mean absolute errors and STD of the errors (b) for Test 4 in the CBD area

Table 5. Statistics of the positioning errors for Test 4 in CBD

\begin{tabular}{ccccc}
\hline Parameters & SPP & L1 SBAS & DFMC SBAS & PPP \\
\hline Mean abs. error (East) (m) & 1.18 & 0.89 & 0.85 & 0.71 \\
\hline Mean abs. error (North) (m) & 1.88 & 1.32 & 0.85 & 0.32 \\
\hline STD (East) (m) & 1.49 & 1.20 & 1.14 & 0.83 \\
\hline STD (North) (m) & 1.80 & 1.47 & 1.22 & 0.49 \\
\hline RMS (East) (m) & 1.59 & 1.20 & 1.16 & 0.85 \\
\hline RMS (North) (m) & 2.33 & 1.64 & 1.23 & 0.50 \\
\hline Availability (solutions) & $70 \%$ & $81 \%$ & $81 \%$ & $71 \%$ \\
\hline Outlier rate & $5 \%$ & $6 \%$ & $5 \%$ & $3 \%$ \\
\hline
\end{tabular}




\section{Test 5: Results of the Mine site}

The last test was performed at a mine site in the Pilbara region, Western Australia in November 4 2018, where the GNSS receiver was fitted on a drill machine. The test time interval was from 07:10:39 to 07:34:34 in GPST. As shown in Figure 6a, the SPP and SBAS solutions have all shown systematic effects caused possibly by multipath. The PPP solutions were experiencing convergence, and some of them at the beginning were removed during outlier detection. Based on Figure 6 b and Table 6 , the SBAS-aided solutions provide better precision and accuracy than the SPP solutions. The PPP solutions have shown the best positioning behaviours with standard deviations and RMS below 3 deci-meter, taking all valid epochs after outlier detection into consideration, including also those before convergence. The availabilities of the SBAS solutions have reached $100 \%$. The DFMC SBAS performance in terms of all metrics were significantly better than those of L1 SBAS.

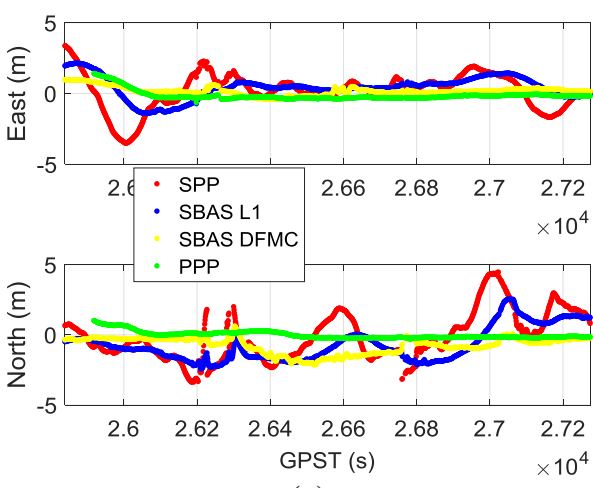

(a)
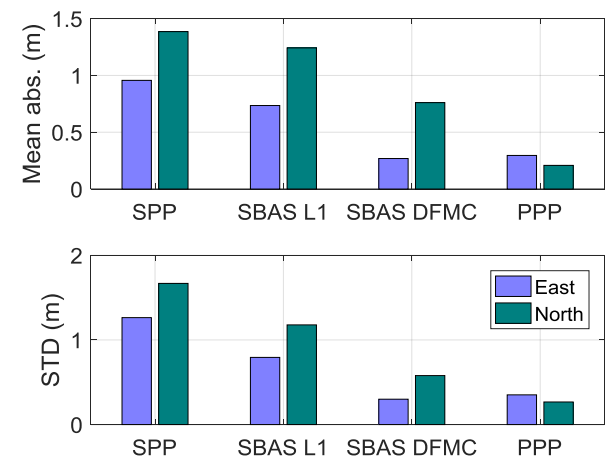

(b)

Fig 6. East and north positioning errors (a), the mean absolute errors and STD of the errors (b) for Test 5 at the mine site

Table 6. Statistics of the positioning errors for Test 5 in at mine site

\begin{tabular}{ccccc}
\hline Parameters & SPP & L1 SBAS & DFMC SBAS & PPP \\
\hline Mean abs. error (East) $(\mathrm{m})$ & 0.96 & 0.74 & 0.27 & 0.30 \\
\hline Mean abs. error (North) $(\mathrm{m})$ & 1.39 & 1.24 & 0.76 & 0.21 \\
\hline STD (East) $(\mathrm{m})$ & 1.26 & 0.79 & 0.30 & 0.35 \\
\hline STD (North) $(\mathrm{m})$ & 1.67 & 1.18 & 0.58 & 0.27 \\
\hline RMS (East) (m) & 1.28 & 0.89 & 0.35 & 0.38 \\
\hline RMS (North) (m) & 1.68 & 1.38 & 0.95 & 0.27 \\
\hline Availability (solutions) & $100 \%$ & $100 \%$ & $100 \%$ & $100 \%$ \\
\hline Outlier rate & $0 \%$ & $0 \%$ & $0 \%$ & $6 \%$ \\
\hline
\end{tabular}

\section{BRIDGING POSITIONING DURING SBAS OUTAGES IN THE URBAN ENVIRONMENT}

It is evident from our tests that, unlike the application of SBAS in aviation where SBAS is almost available all the time, in transportation and land applications SBAS signals may occasionally suffer from outages due to being obstructed by structures depending on geometry of surrounding structures and direction of SBAS line of sight. Therefore, the second part of the paper proposes a method to bridge such short outage in the SBAS orbit and clock corrections, which are auto-regressively predicted as time series based on $1 \mathrm{hr}$ of stored data before occurrence of the outage. We focus here on the new DFMC SBAS technique and SBAS-based PPP. The same principles can be applied to predict the orbit and clock corrections of L1 SBAS, however, the ionosphere corrections for L1 SBAS are not predicted and the application of the last saved values should be limited to a few minutes.

For short outages of less than $15 \mathrm{~min}$ in the orbit corrections, i.e. the differences between the broadcast and precise orbits, are predicted as a time series using a Holt-Winters' auto-regressive model. The Holt-Winters' method comprises a level component, a trend component and a component representing seasonal variations [11]. Initial values for the level and trend components are obtained from a linear regression on time, whereas initial values for the seasonal component are obtained from a dummy-variable regression using de-trended data. Next, weights are used to update the three components, where the smoothing equations of the multiplicative model are expressed as [12]: 


$$
\begin{aligned}
& L_{t}=\alpha \frac{d s_{t}}{S_{t-p}}+(1-\alpha)\left(L_{t-1}+T_{t-1}\right) \\
& T_{t}=\gamma\left(L_{t}-L_{t-1}\right)+(1-\alpha) T_{t-1} \\
& S_{t}=\beta \frac{d s_{t}}{L_{t}}+(1-\beta) S_{t-p} \\
& \delta \hat{\rho}=\left(L_{t-1}+T_{t-1}\right) S_{t-p}
\end{aligned}
$$

where $L_{t}, T_{t}$ and $S_{t}$ are the level, trend, and seasonal components at time $t$, which are determined using least squares from $1 \mathrm{hr}$ of stored orbit corrections prior to the outage. $p$ is the seasonal period. $\alpha, \gamma$ and $\beta$ denote their weights, taken empirically $\alpha=\gamma=0.2$ and $\beta=0.5$. The process is performed every 15 minutes. Figure 7 demonstrates the performance of this orbit prediction method through one representative example using SBAS data. The figure illustrates the prediction error using this approach in terms of the 3D difference between the predicted orbit corrections and their original values for five GPS Satellites. As the Figure shows, the prediction error was less than $10 \mathrm{~cm}$ for the first 5 minutes, and mostly within $20 \mathrm{~cm}$ after 15 minutes of prediction. Another method that can be used is to utilize the predicted part of the most recent IGS ultra-rapid orbits (IGU). This approach though requires downloading this product, and it is available for GPS only. The IGU is released four times daily. The differences between the IGU (predicted half) and IGS GPS final orbits, which can be considered as a bench mark, are within $\pm 7 \mathrm{~cm}$ with standard deviations of 5 $\mathrm{cm}[6]$. One however has to ensure the consistency of the SBAS and IGU orbits, and check whether the orbit corrections refer to the satellite body center or the antenna phase center (i.e. may need to apply PCO). In addition, a datum shift between the two orbit corrections may be needed. Therefore, in this article this approach was not followed. The IGU's clock corrections are also not used since they are provided with long time intervals and have RMS of almost 3 ns.

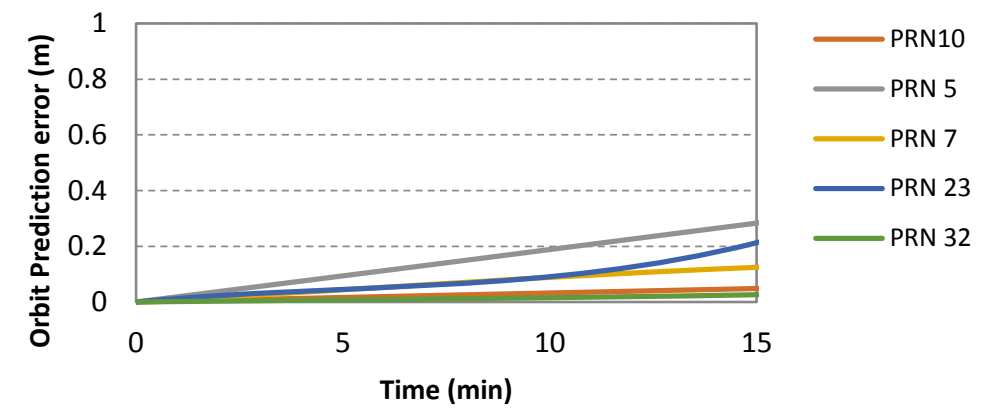

Fig 7. Prediction error of the 3D orbital corrections using Holt-Winters' method

In our work, the clock corrections are predicted using a second order polynomial with sinusoidal components in the form [6]:

$$
\delta t_{\text {predicted }}=a_{0}+a_{1} \Delta T+\sum_{i=0}^{n} A_{i} \sin \left(\frac{\Delta t_{i}}{\lambda_{i}} \times 2 \pi+\frac{t_{\phi_{i}}}{\lambda_{i}} \times 2 \pi\right)+\varepsilon_{\delta t}
$$

where $\delta t_{\text {predicted }}$ denotes the predicted clock correction, $\Delta T$ is the time since start of prediction, $a_{0}$ and $a_{1}$ are the bias and drift of the clock corrections, respectively, and $\varepsilon_{\delta t}$ denotes the noise. $n$ is the number of sinusoidal periods $\left(\lambda_{i}\right)$, which are selected based on spectral analysis of the data using Fast Fourier Transform. $A_{i}$ is the amplitude of period $i, \Delta t_{i}$ denotes the time since start of this period, and $t_{\phi_{i}}$ is its initial phase in time units. These parameters are computed from (5) every 15 minutes using least squares through an estimation period prior to the outage, taken as $1 \mathrm{hr}$, where these parameters $\left(a_{0}, a_{1}, A_{i}\right)$ are treated as the unknowns and the observations are the transmitted SBAS clock corrections ( $\delta t$, placed on the LHS of Equation 5). After determination of these parameters, the model is applied using (5), such that $\delta t_{\text {predicted }}$ is used whenever an SBAS outage is experienced.

The prediction errors for the clock corrections of various GPS and Galileo satellites for one data set collected on 14/9/2018 are illustrated in the Figure 8 as a representative example. The prediction errors are defined as the difference between the actual SBAS clock corrections and their predicted values. The Figure shows GPS clock prediction errors in the left side and Galileo clock prediction errors in the right side. The figure shows errors when prediction is performed for $15 \mathrm{~min}$. The statistics of the errors for the three prediction periods in terms of the mean error, standard deviations and absolute maximum error are given in Table 7. From the Figure 8 and Table 7 one can draw the following conclusions; i) prediction errors of Galileo clock corrections were significantly 
less than those of GPS, indicating more stability of the clock corrections of Galileo compared to GPS, with mean values of about $0.12 \mathrm{~ns}$ for the former and $0.26 \mathrm{~ns}$ for the latter, ii) the prediction error grow slowly with time.
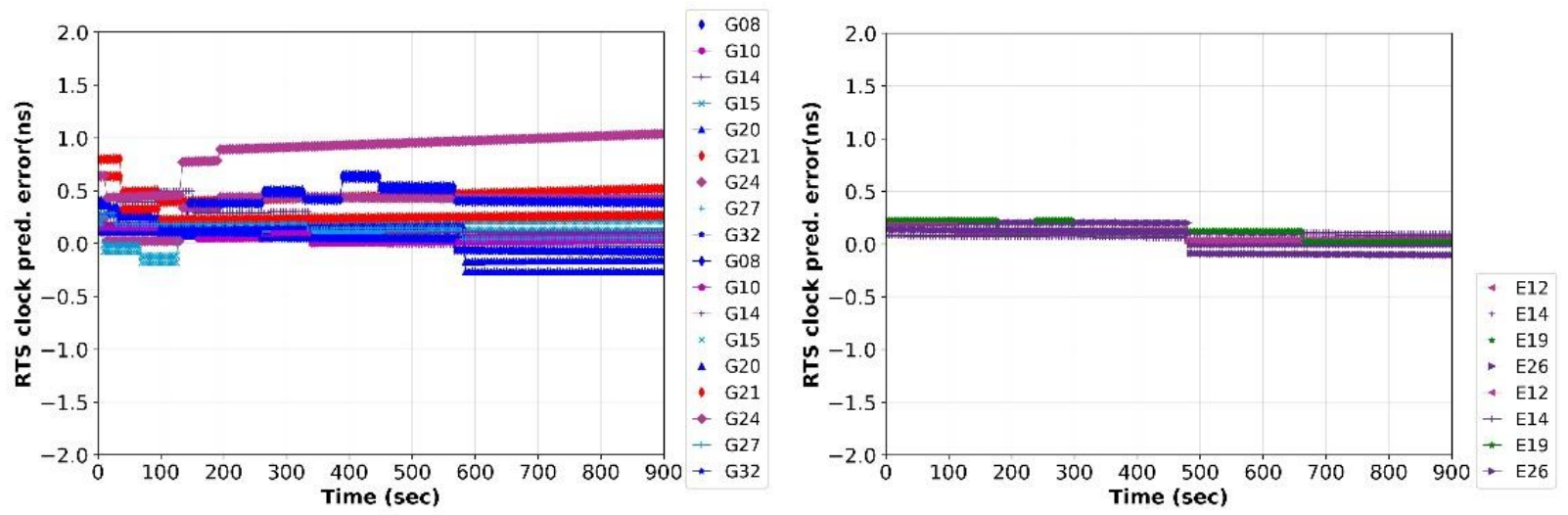

Fig 8. Prediction errors for the clock corrections of GPS (left) and Galileo (right) of all observed satellites at 2:00 GPS time,

Table 7. Statistics of prediction errors of clock corrections

\begin{tabular}{lllll}
\hline System & $\begin{array}{c}\text { Prediction } \\
\text { period }(\mathrm{min})\end{array}$ & $\begin{array}{c}\text { Mean err } \\
(\mathrm{m})\end{array}$ & $\begin{array}{c}\text { Std } \\
(\mathrm{m})\end{array}$ & $\begin{array}{c}\text { abs max_err } \\
(\mathrm{m})\end{array}$ \\
\hline $\mathrm{E}$ & 5 & 0.142 & 0.059 & 0.23 \\
$\mathrm{E}$ & 10 & 0.121 & 0.069 & 0.23 \\
$\mathrm{E}$ & 15 & 0.104 & 0.083 & 0.23 \\
\hline $\mathrm{G}$ & 5 & 0.265 & 0.208 & 0.91 \\
$\mathrm{G}$ & 10 & 0.266 & 0.246 & 0.974 \\
$\mathrm{G}$ & 15 & 0.263 & 0.277 & 1.037 \\
\hline
\end{tabular}

To demonstrate the impact of the prediction error on the positioning results, the positioning errors due to prediction of the orbit and clock corrections are computed, where we process the observation, first with actual SBAS data and next in a separate run with the predicted data for $15 \mathrm{~min}$. The difference between the two position sets represent the position error due to prediction. Figure 9 illustrate this position error for the DFMC SBAS as an example for this period for the East, North, Up coordinates and the overall 3D. As the figure shows, the position error increased with the prediction time and the maximum 3D position error, for example, was $0.26 \mathrm{~m}$ with a mean value of $0.17 \mathrm{~m}$.

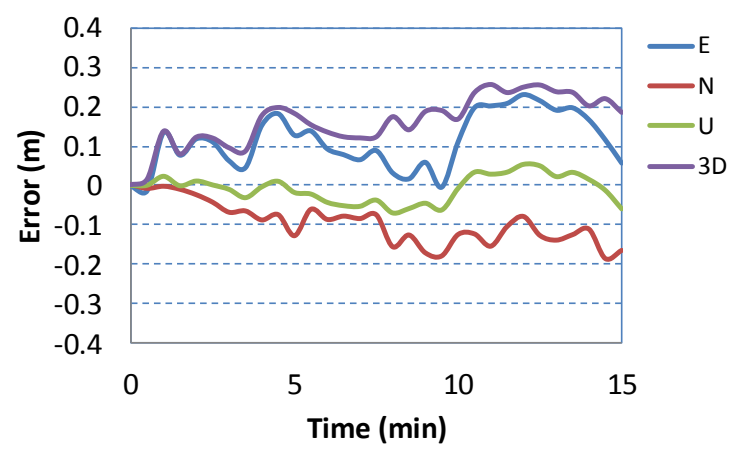

Fig 9. Positioning errors due to prediction the orbit and clock corrections at 2:00 GPS time. 


\section{PROTECTION LEVELS DURING OUTAGES OF THE NEW DFMC SBAS MESSAGES}

In this section, we show the consequences of outage in SBAS on integrity monitoring (IM), in particular for the new DFMC SBAS. IM of L1 SBAS will undergo similar behaviour, therefore, it is sufficient to investigate IM for the new DFMC SBAS, and IM for PPP is still under development, and thus is not discussed here. The horizontal protection levels (HPLs) that bound the horizontal positioning error are only computed, as for most navigation applications on land horizontal positioning is of interest. Availability of positioning integrity monitoring (IM) is judged by checking that HPL < HAL, where HAL is the horizontal alert limit, selected based on the application at hand. For a period of possible outage, the DFMC SBAS HPLs are compared for two cases, the first when using actual SBAS messages over L5 and in the second assuming an outage during the same period and applying the degradation parameters of the message. In this preliminary demonstration, we do not differentiate computation of the HPLs under different urban measurement environments and simply apply the strategies used for aviation [13]. In the DFMC SBAS, the HPLs refer to the protection levels in the direction of the semi-major axis of the horizontal error ellipse, which is formulated as:

$$
\mathrm{HPL}=K \cdot \sigma_{m}
$$

with the factor $K$ calculated from the pre-defined horizontal probability of hazardously misleading information $\left(\mathrm{H}_{\mathrm{PMI}}\right)$, and $\sigma_{m}$ denotes the formal standard deviation of the horizontal positioning error along the semi-major axis of its error ellipse. The terms $K$ and $\sigma_{m}$ can be calculated as:

$$
\begin{gathered}
K=Q^{-1}\left(\frac{\mathrm{H}_{\mathrm{PMI}}}{2}\right) \\
\sigma_{m}=\sqrt{\frac{\sigma_{N}^{2}+\sigma_{E}^{2}}{2}+\sqrt{\left(\frac{\sigma_{E}^{2}-\sigma_{N}^{2}}{2}\right)^{2}+\sigma_{N E}^{2}}}
\end{gathered}
$$

where $Q(\cdot)$ is the one-side tail probability of a standard normal distribution. $\sigma_{N}$ and $\sigma_{E}$ are the formal standard deviations of the north and east positioning errors, and $\sigma_{N E}$ is the covariance element for $N$ and $E . \mathrm{H}_{\mathrm{PMI}}$ of $10^{-4}$ is assumed for the computation in this application. The values of $\sigma_{N}, \sigma_{E}$ and $\sigma_{N E}$ are derived from the variance-covariance matrix of the estimated parameters, denoted by $C_{x}$, which is calculated using least-squares adjustment, expressed as:

$$
C_{x}=\left(A^{T} C_{y}^{-1} A\right)^{-1}
$$

with the design matrix $A=\left[\left[\mu^{1}, \cdots, \mu^{m}\right]^{T}, e_{G}, e_{E}\right]$, where $\mu^{s}$ denotes the satellite-to-receiver unit vector for satellite $s$, and $e_{G}$ and $e_{E}$ correspond to the columns in design matrix for the GPS and Galileo receiver clocks. The element in $e_{G}$ is 1 when the corresponding observation is transmitted from GPS satellites, and amounts to 0 for Galileo satellites, and vice versa for $e_{E}$. With the carriersmoothed ionosphere-free combination of dual-frequency code observations used for the DFMC SBAS scenario, the observations are assumed to be uncorrelated, such that the variance-covariance matrix of the observations $C_{y}$ is a diagonal matrix with the variance element corresponding to satellite $s$ denoted as $\sigma_{s}^{2}$ calculated as follows [13]:

$$
\sigma_{s}^{2}=\sigma_{s, D F C}^{2}+\sigma_{s, l}^{2}+\sigma_{s, \tau}^{2}+\sigma_{s, n}^{2}+\sigma_{s, M P}^{2}
$$

where $\sigma_{S, D F C}$ is the standard deviation of the SBAS correction errors projected onto the observation direction, which is calculated based on the SBAS message according to [13]. $\sigma_{s, l}$ is the standard deviation of the remaining ionospheric residuals, referring to highorder terms not eliminated by the ionosphere-free observation combination, which is modelled elevation-dependent. $\sigma_{s, \tau}$ is the standard deviation of the slant tropospheric residuals after removing the modelled part, which is computed based on a zenithreferenced standard deviation of the tropospheric residuals of $0.12 \mathrm{~m}$ and an elevation-dependent mapping function. $\sigma_{s, n}$ is the noise standard deviation, which is assumed to be bounded by $0.4 \mathrm{~m}$ for the carrier-smoothed DFMC observations. $\sigma_{S, M P}$ is the elevationdependent formal standard deviation of the multipath effects in the carrier-smoothed code observations, which amounts to about 0.34 $\mathrm{m}$ in the vertical direction and $0.84 \mathrm{~m}$ at the elevation mask of 10 degrees. The value of $\sigma_{s, M P}$ is currently borrowed from the aviation applications, and needs to be further refined for different land environments in future studies. 
During outage of the SBAS messages, the receivers cannot receive the up-to-date integrity and correction information from the SBAS satellite anymore. Old corrections and integrity information thus are used instead. Even ignoring the time-out windows for different parameters defined for aviation applications, degradation of the SBAS corrections could take place, which grows with the time of the outage. In addition, as the SBAS corrections have to match their Issue of Data Number (IODN) to the set of broadcast clock and ephemeris having the same IOD Clock (IODC) in the GPS navigation file and the same IOD of the nav batch (IODnav) in the Galileo F/NAV navigation message, using old SBAS corrections may lead to the use of old ephemeris/clocks.

As an example, the HPLs are shown in Figure 10 for the station CUAA located in Curtin University, Perth, Australia. The data between 1:00 and 1:30 in GPS time (GPST) on 14/9/2018 was used for the computation. The HPLs without outage of the SBAS messages are plotted as blue dots, which was about $4 \mathrm{~m}$. Due to the currently limited number of the GPS IIF satellites transmitting L5 signals, the test is performed making use of the GPS L1/L2 and Galileo E1/E5a scenario. About 8 to 9 GPS satellites and 5 to 6 Galileo satellites were observed and used in the processing. Beginning from the epoch 4500, SBAS messages was assumed to be unavailable in one of the test scenarios, and the degraded HPLs from the observed satellites are illustrated as red dots.

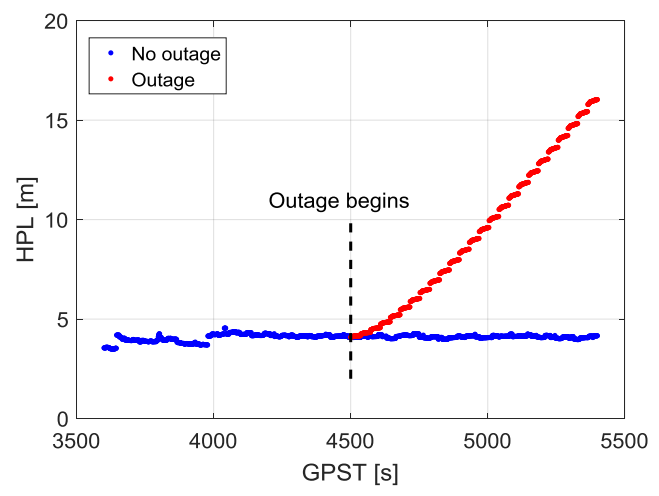

Fig 10. HPLs of the station CUAA with DFMC SBAS messages (blue) and during the assumed SBAS outage (red). The data between 1:00 and 1:30 in GPST, 14/9/2018 was used for the plot

The step-wise degradation of the HPLs from the onset of the assumed outage in Figure 10 is mainly caused by the degradation in the SBAS corrections with time. Figure 11 shows the main parameter in HPL, the $\sigma_{S, D F C}$ (see equation 10), for GPS satellite G02 and Galileo satellite E07 during the same time period. A step-wise degradation of $\sigma_{S, D F C}$ every $36 \mathrm{~s}$ resulted for both satellites in our case. The value of $\sigma_{S, D F C}$ is computed as the square root of the squared sum of the corresponding variance at the time of applicability of the corrections and the variance for degradation, and the variance of degradation is calculated based on the step and the first-order degradation parameters contained in the last recorded DFMC SBAS message before the outage. For an outage of 15 min, the degradation of $\sigma_{S, D F C}$ is up to several meters, and the resulting degradation in HPLs is more than $10 \mathrm{~m}$ after 8 min as shown in Figure 10, and after $15 \mathrm{~min}$ of outage the HPLs were about $17 \mathrm{~m}$. For precise land applications, for instance, intelligent transport systems, such high HPL values are unacceptable for applications that require lane identification.
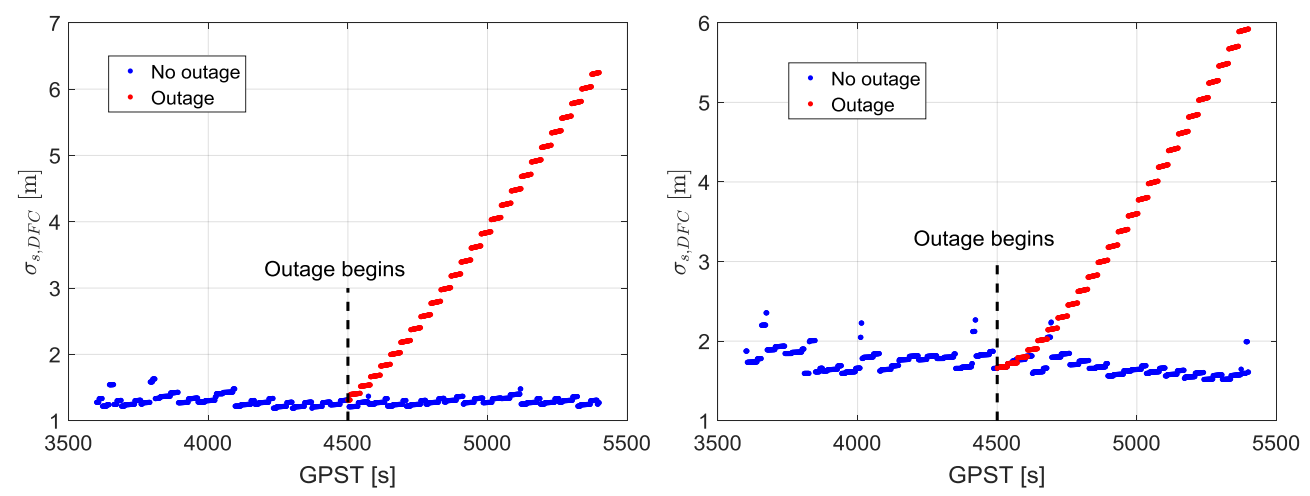

Fig 11. $\sigma_{S, D F C}(5)$ for GPS satellite G02 (left) and Galileo satellite E07 (right) between 1:00 and 1:30 in GPST on 14/9/2018 
In the future, I is expected that advanced generation of GNSS, e.g., GPS Block III satellites, and availability of more Galileo satellites would provide better visibility and signal quality, and thus more precise satellite orbit and clock corrections could be available for SBAS users. In addition to that, the stability of the on-board satellite clocks and the orbit dynamic models continuously improve with time. Thus, smaller degradation parameters could be expected in the future. For illustration, we experiment the impact when the step degradation parameters that were available during the processing time period are halved as expected in the future (see Figure 10), and the HPLs were recomputed. Their values are depicted in Figure 12. In this case, the degradation of the HPLs is reduced to about $6 \mathrm{~m}$, and the HPL after an outage of $15 \mathrm{~min}$ is lower than $10 \mathrm{~m}$.

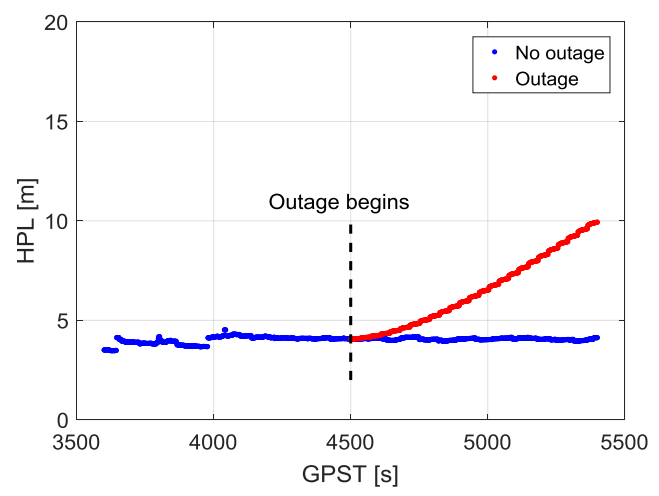

Fig 12. HPLs of the station CUAA with DFMC SBAS messages (blue) and during the assumed SBAS outage (red) using half of the step degradation parameters of current SBAS corrections. The data between 1:00 and 1:30 in GPST on on 14/9/2018 was used.

When experience SBAS outage, IM can be performed using Advanced Receiver Autonomous Integrity Monitoring (ARAIM) [14]. However, the method parameters have to be consistent with DFMC SBAS IM assumptions for coherency of the service, and adapt to land applications in the urban environment. In addition, several algorithm modifications is needed for application of the IM in DFMC SBAS in this environment. For instance, the observations that experience large multipath should be detected and deweighted [15]. Hence, adjusting the value of $\sigma_{s, M P}$ to the local multipath conditions. One possible way here is to use 3D city models to detect which surface of the surrounding buildings could make a specular reflection. In addition, due to the presence of multipath and No line-of-sight (NLOS) in the land applications, the distribution of observation errors might not be exactly Gaussian, and have multiple beaks and its mean could be biased from zero, therefore, the overbounding concept [16], could be implemented and tuned for this application. These issues will be discussed for DFMC SBAS in a follow-up paper.

\section{CONCLUSIONS}

The paper comprises three sections. The first section presents performance analysis of the AUS/NZ second generation SBAS (L1, DFMC) and SBAS-based PPP positioning for transport applications and in mining. Testing in open-sky environment shows that the solution availability was above $95 \%$ and the performance of L1 SBAS is similar to DFMC SBAS, with mean error close to $1 \mathrm{~m}$, which was better than the SPP. The PPP solutions have smaller error range of $0.15 \mathrm{~m}$ on average. In the low-density urban area, positioning availability dropped to about $80 \%$, and position errors almost doubled for all three modes due to observing a less number of satellites, leading to a weaker model, and degraded observation quality due to the presence of multipath and NLOS signals. In the high-density urban environment in the CBD area, results further degraded. In the mine environment, which is open to sky but is affected by multipath, sub-m positioning accuracy was obtained for the L1 SBAS and DFMC modes and $0.2 \mathrm{~m}$ for PPP. Overall DFMC SBAS had slightly better performance than L1 SBAS.

The second part of the paper proposes a method to bridge positioning during short outages in SBAS, which may take place in the urban environment due to SBAS signal blockage. The orbit and clock corrections were predicted as a time series. For short outages less than $15 \mathrm{~min}$ in the orbit corrections, these corrections were predicted using a Holt-Winters' auto-regressive model. Prediction error less than $10 \mathrm{~cm}$ was achieved after the first $5 \mathrm{~min}$, and about $20 \mathrm{~cm}$ after 15 min of prediction. The clock corrections were predicted using a second order polynomial with sinusoidal components. Results showed that prediction errors of Galileo clock corrections were significantly less than those of GPS, with mean values of about $0.12 \mathrm{~ns}$ for Galileo and $0.26 \mathrm{~ns}$ for GPS, and the prediction error grow slowly with time. 
The third section of the paper shows the impact of these DFMC SBAS outages on computation of the horizontal protection levels needed for integrity monitoring. Using the last received SBAS message, a step-wise degradation of the HPLs from the onset of the assumed outage is shown, caused by the degradation in the SBAS parameters with time. A numerical example shows that with the SBAS outage, the degradation of $\sigma_{S, D F C}$ is up to several meters, resulting in HPLs of more than $10 \mathrm{~m}$ after $8 \mathrm{~min}$, and after $15 \mathrm{~min}$ of outage the HPLs were about $20 \mathrm{~m}$. Therefore, methods such as ARAIM can be used for integrity monitoring during the SBAS outage. However, it has to be consistent with DFMC SBAS. This will be addressed in our future research, which will also consider deweighting the observations suffering from multipath through a representative model and consider an overbounding distribution, or models that better describe the distribution of the observation errors, which might not be Gaussian in the urban environment.

\section{ACKNOWLEDGMENTS}

FrontierSI and Geoscience Australia are acknowledged for their support and funding of SBAS test-bed PD8703 and PD8602 projects, where some of their results are used in this study. The authors would like to thank Transport for New South Wales (NSW) and Roy Hill for realizing the experiments at NSW and the Roy Hill mine site. Norman Cheung, Joon Wyan and David Imparato are recognized for their help in data collection in the NSW tests. Manoj Deo is acknowledged for processing some of the data used.

\section{REFERENCES}

1. Sobreira, H., Bougard, B., Barrios, J., Calle, J.D., "SBAS Australian-NZ Test Bed: Exploring New Services", Multi GNSS Asia Conference. Melbourne, Australia. 23-25 October 2018.

2. Minimum Operational Performance Specification for Galileo / Global Positioning System /Satellite-Based Augmentation System Airborne Equipment; DFMC SBAS L5 MOPS Draft WG62_GAL_GPS_SBÅS_MOPS_v0.6.1_21_Jun_2018.

3. Minimum Operational Performance Specification for Galileo / Global Positioning System /Satellite-Based Augmentation System Airborne Equipment; DFMC SBAS L5 MOPS Draft WG62_GAL_GPS_SBAS_MOPS_v0.3.8_10_Mar_2017.

4. Zumberge, J.F., Heftin, M.B., Jefferson, D.C., Watkins, M.M. and Webb, F.H., "PPP for the efficient and robust analysis of GPS data from large networks", J Geo.Res., Vol 102, No. B3, 1997, pp. 5005-5017.

5. Heroux, P., Gao, Y., Kouba, J., Lahaye, F., Mireault, Y., Collins, P., Macleod K., Tetreault, P., Chen, K., "Products and Applications for Precise Point Positioning - Moving Towards Real-Time, Proc. of the ITM of the ION GNSS, Long Beach, CA, Sept $21-24,2004$, pp $1832-1843$.

6. El-Mowafy, A., Deo, M., Kubo, N., "Maintaining real-time precise point positioning during outages of orbit and clock corrections", GPS Solutions, Vol. 21, No. 3, 2017, pp. 937-947.

7. Barrios, J., Caro, J., Calle, J.D., Carbonell, E., Pericacho, J.G., Fernández, G., Esteban, V.M., Fernández, M.A., Bravo, F., Torres, B., Calabrese, A., Diaz, A., Rodríguez, I., Laínez, M.D., Romay, M.M., Jackson, R., Reddan, P.E., Bunce, D., Soddu, C., "Update on Australia and New Zealand DFMC SBAS and PPP System Results", In Proceedings of ION GNSS+ 2018, Miami, Florida, September 24-28, 2018, pp. 1038-1067.

8. Cogdell, K., Reddan, P., "Australia/New Zealand DFMC SBAS and Navigation Message Authentication", In Proceedings of ION GNSS+ 2018, Miami, Florida, September 24-28, 2018, pp. 1068-1083.

9. El-Mowafy, A., "Real-Time Precise Point Positioning Using Orbit and Clock Corrections as Quasi-Observations for Improved Detection of Fault", J. of Navigation, Vol. 71, No. 4, 2018, pp. 769-787.

10. Technical Specifications (2017) Technical Specifications Document for Satellite-Based Augmentation System (SBAS) Testbed. Revision 5, November 10, 2017.

11. Chatfield C, Yar M., "Prediction Intervals for Multiplicative Holt-Winters", International Journal of Forecasting, Vol. 7, 1991,pp. 31-37.

12. El-Mowafy, A. "Predicting Real-Time Orbit and Clock Corrections for Positioning Using GPS, GLONASS and QZSS in Natural Hazard Warning Systems", Journal of Applied Geodesy, Vol., 13, no. 2, 2019, pp. 69-79.

13. EUROCAE. "Minimum operational performance standard for Galileo / Global Positioning System / Satellite-based augmentation system airborne equipment". The European organisation for civil aviation equipment, ED-259, February 2019.

14. Blanch, J, Walter, T, Enge, P, Lee, Y, Pervan, B, Rippl, M, Spletter, A, Kropp, V, "Baseline advanced RAIM user algorithm and possible improvements", IEEE Transactions on Aerospace and Electronic Systems. Vol 51, No. 1, 2015, pp 713-732.

15. Pirsiavash, A, Broumandan, A, Lachapelle, G, O'Keefe, K, "Detection and De-weighting of Multipath-affected Measurements in a GPS/Galileo Combined Solution", In Proceedings of the European Navigation Conference 2019, 9-12 April, Warsaw, Poland, pp. 1-12.

16. Rife, J, Pullen, s, Enge, P, Pervan, B, "Paired Overbounding for Nonideal LAAS and WAAS Error Distributions", IEEE Trans. On Aerospace and Electronic Systems, Vol. 42, No. 4, 2006, pp. 1386-1395. 